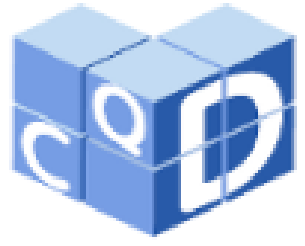

Revista Eletrônica

Paulista de Matemática

ISSN 2316-9664

Volume 11, dez. 2017

Francisco Regis Vieira Alves Instituto Federal de Educação, Ciência e Tecnologia do Estado do Ceará - IFCE.

fregis@ifce.edu.br

\author{
Rannyelly Rodrigues de \\ Oliveira \\ Instituto Federal de Educação, \\ Ciência e Tecnologia do Estado \\ do Ceará - IFCE. \\ nanny-rockstar@hotmail.com
}

\section{Sobre o modelo de Fibonacci na variável complexa: identidades generalizadas}

\author{
On the complex variable Fibonacci's model: generalized
} identities

\begin{abstract}
Resumo
A sequência emblemática relacionada com o problema da reprodução de pares de coelhos, propugnado por Leonardo Pisano, em 1202, se tornou, passados alguns séculos, o objeto de estudo e pesquisa em determinados ramos particulares da Matemática, sobretudo a partir dos anos 60. As repercussões podem ser constadas a partir de uma profusão de modelos e formas de sua generalização. Isso posto, no presente trabalho, lidamos com as formas polinomiais de representação do modelo Generalizado de Fibonacci na variável complexa. Assim, por intermédio de engenhosas representações matriciais, deduzimos três identidades clássicas e intimamente vinculadas ao modelo de Fibonacci. Ademais, na última seção, trazemos uma nova definição derivada ainda do modelo na variável complexa. Por fim, as respectivas identidades são descritas ainda para um conjunto maior de índices, caracterizando um processo de sua extensão e generalização.
\end{abstract}

Palavras-chave: Sequência de Fibonacci. Variável complexa. Identidades generalizadas.

\begin{abstract}
The emblematic sequence related to the problem of the reproduction of pairs of rabbits proposed by Leonardo Pisano, in 1202 became, after a few centuries, the object of study and research in certain particular branches of Mathematics, from the sixties. The repercussions can be seen from a profusion of models and forms of their generalization. This, in the present work, we deal with the polynomial forms of representation of the Generalized Fibonacci model, in the complex variable. Thus, through some ingenious matrix representations, three classical identities, closely linked to the Fibonacci's model are deduced. In addition, in the last section, we bring a new definition derived from the complex variable's model. Finally, their identities are further described for a larger set of subscritps, characterizing a process of their extension and generalization.
\end{abstract}

Keywords: Fibonacci’s sequence. Complex variable. Generalized identities. 


\section{Introdução}

Recorrentemente, na literatura especializada, reconhecemos um modelo matemático de uma sequência homogênea recorrente de segunda ordem, por intermédio de um lacônico problema formulado por Leonardo Pisano em 1202. Por outro lado, a partir dos anos 60, registramos alguns trabalhos envolvendo uma preocupação com o aspecto histórico e evolutivo da sequência de Fibonacci [1, 2, 3, 4, 5], ou seja, a sua generalização [6, 7, 8, 9, 10, 11] com ênfase no processo de complexificação do modelo de Fibonacci, isto é, da introdução de unidades imaginárias, do aumento dimensional e a correspondente propriedade das representações dos números de Fibonacci ou, dizendo melhor, de números complexos de Fibonacci.

Nesse sentido, a despeito da descrição das propriedades do modelo de Fibonacci na variável complexa, trazemos ainda a dedução de algumas identidades clássicas, largamente discutidas em alguns livros, todavia, com a sua descrição correspondente para índices naturais. Dessa forma, recordamos que a identidade descoberta em 1680 pelo matemático astrônomo italiano Giovani Domenico Cassini (1625 - 1712) e de modo independente, em 1753, estudada pelo matemático escocês Robert Simson $(1687-1768)$, é conhecida por $f_{n+1} f_{n-1}-f_{n} \cdot f_{n}=(-1)^{n}$, para $n \geq 0$.

A generalização dessa identidade, em 1879, foi devida ao trabalho do matemático belga Eugène Charles Catalan (1814 - 1894), indicada por $f_{n} f_{n}-f_{n+r} f_{n-r}=(-1)^{n-r} f_{r}^{2}$, para $m \geq n \geq 1$ . Outra forma de sua generalização foi consequência do trabalho do engenheiro e matemático francês Philbert Maurice d'Ocagne (1862 - 1938), que nasceu em Paris em 1862 e, além de outros trabalhos sobre Cálculo e Geometria, deduziu ainda a seguinte expressão $f_{m+1} f_{n}-f_{m} f_{n+1}=(-1)^{m} f_{n-m}$, para $m \geq n \geq 1$. Por fim, não nos furtamos de prestar uma pequena homenagem ao matemático canadense Ross Honsberger (1929 - 2016), cuja identidade $f_{n+m}=f_{n-1} f_{m}+f_{n} f_{m+1}$, para $m \geq n \geq 0$, formulada em [7] inspirou inúmeras outras derivações [12].

\section{Representação polinomial do modelo de Fibonacci}

A tradição dos estudos que investigam propriedades das representações polinomiais, em alguns casos com a introdução de um parâmetro, de uma variável ou várias variáveis possui um marco bastante representativo na década de 70. Assinalamos alguns trabalhos pioneiros $[6,8$, $9,12,13,14,15]$ que concorreram para uma profusão de repercussões e implicações em outros ramos de pesquisa intimamente relacionados com o modelo da Sequência Generalizada de Fibonacci.

Os polinômios de Fibonacci foram estudados pela primeira vez em 1883 pelo matemático belga Eugene Charles Catalan (1814 - 1894) e matemático alemão Ernest Erich Jacobsthal (1881 - 1965). Assim, Catalan definiu a seguinte família de funções polinomiais de Fibonacci:

Definição 1. Chamaremos a Sequência Polinomial de Fibonacci (SPF), o conjunto de funções polinomiais descrito pela relação de recorrência:

$$
f_{n}(x)=x \cdot f_{n-1}(x)+f_{n-2}(x), \operatorname{com} f_{1}(x)=1, f_{2}(x)=x, \text { e } n \geq 1 \text {. }
$$

Sem dúvida, através da definição anterior, podemos perceber o processo de generalização que inicialmente ocorreu com a sequência de Fibonacci e, gradualmente, após algumas décadas, começou a ser registrado em vários outros modelos de sequências generalizadas. Tal repercussão evidencia o caráter de ubiquidade do modelo de Fibonacci. Assim, na próxima

ALVES, F. R. V.; OLIVEIRA, R. R. de. Sobre o modelo de Fibonacci na variável complexa: identidades generalizadas. C.Q.D.- Revista Eletrônica Paulista de 
seção, estudaremos as relações recursivas decorrentes da consideração da variável complexa e que, em maior ou em menor substância, argumentos semelhantes podem ser registrados em outros trabalhos semelhantes.

\subsection{Representação na variável complexa}

De acordo com [16], temos as seguintes definições:

Definição 2. A sequência Generalizada Polinomial (SGP) $\left\{F_{n}(a, z)\right\}_{n=0}^{\infty}$ nas variáveis $a$ e $z$, é definida pela relação de recorrência de segunda ordem:

$$
F_{n}(a, z)=a \cdot \mathbf{z} \cdot F_{n-1}(a, z)+a^{2} \cdot F_{n-2}(a, z), \text { para } n \geq 2
$$

com a seguinte condição inicial $F_{0}(a, z)=0, F_{1}(a, z)=1$. Logo em seguida e de modo particular, consideremos ainda mais uma definição:

Definição 3. A Sequência Polinomial de Fibonacci (SPF), na variável $z$ para $a=1$, é descrita pela seguinte relação de recorrência:

$$
f_{n}(z)=\mathrm{z} \cdot f_{n-1}(z)+f_{n-2}(z), \text { para } n \geq 2
$$

com os seguintes valores iniciais $f_{0}(z)=0, f_{1}(z)=1$. Com origem nas definições anteriores, vamos enunciar o seguinte lema:

Lema 1. ([16]) Para $n \geq 1$, vale a seguinte relação:

$$
F_{n}(a, \mathrm{z})=a^{n-1} \cdot f_{n}(z)
$$

Demonstração: para $F_{1}=1=a^{0} \cdot 1=\mathrm{a}^{1-1} \cdot f_{1}(z)$ e $F_{2}=a z=a^{2-1} \cdot z=a^{2-1} \cdot f_{2}(z)$. Recorrendo às definições 2 e 3, a demonstração segue por indução matemática sobre $n$ observando que vale a igualdade $F_{n}=a \cdot z \cdot F_{n-1}+a^{2} \cdot F_{n-2}=a \cdot z \cdot a^{n-2} \cdot f_{n-1}(z)+a^{2} \cdot a^{n-3} \cdot f_{n-2}(z)=a^{n-1}\left(z \cdot f_{n-1}(z)+f_{n-2}(z)\right)=a^{n-1} \cdot f_{n}(z)$, tendo em vista que $F_{n-1}=a^{n-2} \cdot f_{n-1}(z)$ e $F_{n-2}=a^{n-3} \cdot f_{n-2}(z)$.

Dessa forma, pelo lema anterior, determinamos uma relação entre a SGP e a SPF. Por outro lado, tomando a importante noção de função geradora discutida em [10], definida por:

$$
g_{F}(t)=\sum_{n=0}^{\infty} F_{n}(a, z) \cdot t^{n}
$$

assim, temos o seguinte teorema:

Teorema 1. ([16]) A função geradora da SGP é dada por:

$$
g_{F}(t)=\frac{t}{1-a z \cdot t-a^{2} \cdot t^{2}}
$$


Demonstração: a partir da relação de recorrência $F_{n}-a \cdot z \cdot F_{n-1}-a^{2} \cdot F_{n-2}=0$ e da seguinte soma formal $g_{F}(t)=\sum_{n=0}^{\infty} F_{n} \cdot t^{n}$, vamos efetuar as seguintes operações. Primeiramente, vamos façamos o produto $(a z \cdot t) g_{F}(t)=(a z \cdot t) \sum_{n=0}^{\infty} F_{n} \cdot t^{n}$. Logo em seguida, efetuamos ainda o seguinte produto indicado $\left(a^{2} \cdot t^{2}\right) g_{F}(t)=\left(a^{2} \cdot t^{2}\right) \sum_{n=0}^{\infty} F_{n} \cdot t^{n}$. Com efeito, obteremos que:

$$
\left\{\begin{array}{l}
g_{F}(t)=\sum_{n=0}^{\infty} F_{n} \cdot t^{n} \\
(a z \cdot t) g_{F}(t)=a z \cdot \sum_{n=0}^{\infty} F_{n} \cdot t^{n+1} \\
\left(a^{2} \cdot t^{2}\right) g_{F}(t)=a^{2} \cdot \sum_{n=0}^{\infty} F_{n} \cdot t^{n+2}
\end{array}\right.
$$

Logo em seguida, vamos considerar a seguinte expressão $\left(g_{F}(t)-a z \cdot t, g_{F}(t)-a^{2} \cdot t^{2} \cdot g_{F}(t)\right)$, para:

$$
\left\{\begin{array}{l}
g(t):=\sum_{n=0}^{\infty} F_{n} \cdot t^{n}=F_{0} \cdot t^{0}+F_{1} \cdot t^{1}+F_{2} \cdot t^{2}+\cdots+F_{n} \cdot t^{n}+\cdots \\
a z t \cdot g(t):=a z \cdot \sum_{n=0}^{\infty} F_{n} \cdot t^{n+1}=a z \cdot F_{0} \mathrm{t}+a z \cdot F_{1} \cdot t^{2}+\cdots+a z \cdot F_{n-1} \cdot t^{n}+a z \cdot F_{n} \cdot t^{n+1}+\cdots \\
a^{2} t^{2} \cdot g(t):=a^{2} \cdot \sum_{n=0}^{\infty} F_{n} \cdot t^{n+2}=a^{2} \cdot F_{0} \cdot t^{2}+a^{2} \cdot F_{1} \cdot t^{3}+\cdots+a^{2} \cdot F_{n-1} t^{n+1}+a^{2} F_{n} \cdot t^{n+2}+\cdots
\end{array}\right.
$$

Assim, devemos o seguinte $\left(g_{F}(t)-a z \cdot t . g_{F}(t)-a^{2} \cdot t^{2} \cdot g_{F}(t)\right)=F_{0}+t . F_{1}-a z . t . F_{0}+$ $+\sum_{n=2}^{\infty}\left(F_{n}-a z \cdot F_{n-1}-a^{2} \cdot F_{n-2}\right) t^{n}=F_{0}+t F_{1}-a z . t . F_{0}+\sum_{n=2}^{\infty}(0) \cdot t^{n}=t . \quad$ Portanto, $\quad$ segue $\quad$ que $g_{F}(t)=\frac{t}{1-a z \cdot t-a^{2} \cdot t^{2}}$, para $t \in I R$

Por intermédio da teoria das equações recorrentes homogêneas, devemos determinar a seguinte equação característica $t^{2}-a z \cdot t-a^{2}=0$. De imediato, podemos determinar as raízes da seguinte forma $\alpha(a, z)=\frac{a z+a \sqrt{z^{2}+4}}{2}, \beta(a, z)=\frac{a z-a \sqrt{z^{2}+4}}{2}$. Facilmente, podemos ver que $\alpha+\beta=\mathrm{az}, \alpha \cdot \beta=-a^{2}, \alpha-\beta=a \sqrt{z^{2}+4}$. Com origem nessas relações, vejamos outros teoremas desta seção:

Teorema 2. ([16]) Para $n \geq 0$, temos que: 


$$
F_{n}(a, z)=\frac{\alpha^{n}(a, z)-\beta^{n}(a, z)}{\alpha(a, z)-\beta(a, z)}
$$

Demonstração: ademais, de acordo com os argumentos anteriores, garantimos as seguintes relações $\alpha^{2}-a z \cdot \alpha-a^{2}=0, \beta^{2}-a z \cdot \beta-a^{2}=0$. Logo em seguida, vamos recordar a relação fundamental. Assim, pela hipótese indutiva, escrevemos ainda a seguinte relação (determinada por definição): $F_{n}=a \cdot \mathrm{z} \cdot F_{n-1}+a^{2} \cdot F_{n-2}=F_{n+1}=a \cdot \mathrm{z} \cdot F_{n}+a^{2} \cdot F_{n-1}=$ $=a z \cdot \frac{\left(\alpha^{n}-\beta^{n}\right)}{\alpha-\beta}+a^{2} \cdot \frac{\left(\alpha^{n-1}-\beta^{n-1}\right)}{\alpha-\beta}=a z \cdot \frac{\left(\alpha^{n}-\beta^{n}\right)}{\alpha-\beta}+a^{2} \cdot \frac{\left(\frac{\alpha^{n}}{\alpha}-\frac{\beta^{n}}{\beta}\right)}{\alpha-\beta}=a z \cdot \frac{\left(\alpha^{n}-\beta^{n}\right)}{\alpha-\beta}+a^{2} \cdot \frac{\left(\alpha^{n} \beta-\beta^{n} \alpha\right)}{\alpha \beta \cdot(\alpha-\beta)}=a z \cdot \frac{\left(\alpha^{n}-\beta^{n}\right)}{\alpha-\beta}+$ $a^{2} \cdot \frac{\left(\alpha^{n} \beta-\beta^{n} \alpha\right)}{\left(-\mathrm{a}^{2}\right)(\alpha-\beta)}$. Por outro lado, efetuando algumas multiplicações, devemos determinar que são válidas as igualdades $\alpha^{n+1}=a z \cdot \alpha^{n}+a^{2} \cdot \alpha^{n-1}, \beta^{n+1}=a z \cdot \beta^{n}+a^{2} \cdot \beta^{n-1}$. Assim, vemos ainda que:

$$
\begin{aligned}
& F_{n+1}=\frac{a z \cdot \alpha^{n}-a z \cdot \beta^{n}}{\alpha-\beta}-\frac{\alpha^{n-1} \cdot \alpha \beta-\beta^{n-1} \cdot \alpha \beta}{\alpha-\beta}=\frac{a z \cdot \alpha^{n}-a z \cdot \beta^{n}}{\alpha-\beta}-\frac{\alpha^{n-1} \cdot\left(-a^{2}\right)-\beta^{n-1} \cdot\left(-a^{2}\right)}{\alpha-\beta}= \\
& =\frac{a z \cdot \alpha^{n}+\mathrm{a}^{2} \alpha^{n-1}-\left(a z \cdot \beta^{n}+a^{2} \beta^{n-1}\right)}{\alpha-\beta}=\frac{\alpha^{n+1}-\beta^{n+1}}{\alpha-\beta}, \text { para } n \geq 1 .
\end{aligned}
$$

Teorema 3. Para todo inteiro $n$, temos que:

$$
F_{-n}(a, \mathrm{z})=\frac{1}{(-1)^{n+1} a^{2 n}} F_{n}(a, \mathrm{z})
$$

Demonstração: a partir da fórmula de Binnet que demonstramos no teorema anterior, vem que $F_{-n}=\frac{\alpha^{-n}-\beta^{-n}}{\alpha-\beta}=\frac{\left(\frac{1}{\alpha^{n}}-\frac{1}{\beta^{n}}\right)}{\alpha-\beta}=\frac{1}{(\alpha \cdot \beta)^{n}} \cdot \frac{\beta^{n}-\alpha^{n}}{\alpha-\beta}=\frac{1}{\left(-a^{2}\right)^{n}} \cdot \frac{\beta^{n}-\alpha^{n}}{\alpha-\beta}=\frac{1}{(-1)^{n+1} a^{2 n}} \cdot \frac{\alpha^{n}-\beta^{n}}{\alpha-\beta}=$ $=\frac{1}{(-1)^{n+1} a^{2 n}} F_{n}$, para inteiro $n$.

Observamos que, a partir dos resultados passados, quando lidamos apenas com a variável complexa $z$ e fazendo $a=1$, podemos ainda determinar a fórmula de Binnet na variável complexa e a correspondente extensão ao campo de índices inteiros. Ou seja, $f_{n}(\mathrm{z})=\frac{\alpha^{n}(\mathrm{z})-\beta^{n}(\mathrm{z})}{\alpha(\mathrm{z})-\beta(\mathrm{z})}$ e $F_{-n}=\frac{1}{(-1)^{n+1} a^{2 n}} F_{n}$. Ou simplesmente, pelo lema 1 , sabemos que é válida a igualdade $F_{n}=a^{n-1} \cdot f_{n}(z)$, para $n \geq 1$. A partir disso, escrevemos $F_{n}=\frac{\alpha^{n}-\beta^{n}}{\alpha-\beta}=a^{n-1} \cdot f_{n}(z) \Leftrightarrow f_{n}(z)=\frac{1}{a^{n-1}} \cdot \frac{\alpha^{n}-\beta^{n}}{\alpha-\beta}$. 
Antes de concluirmos a presente seção, vamos considerar as seguintes representações matriciais $A^{1}=\left(\begin{array}{ll}a z & 1 \\ a^{2} & 0\end{array}\right)=\left(\begin{array}{cc}F_{2} & F_{1} \\ a^{2} F_{1} & a^{2} F_{0}\end{array}\right)$ e $A^{n}=\left(\begin{array}{cc}F_{n+1} & F_{n} \\ a^{2} F_{n} & a^{2} F_{n-1}\end{array}\right)$, por indução matemática, basta ver que $A^{n+1}=A^{n} \cdot A=\left(\begin{array}{cc}F_{n+1} & F_{n} \\ a^{2} F_{n} & a^{2} F_{n-1}\end{array}\right)\left(\begin{array}{ll}a z & 1 \\ a^{2} & 0\end{array}\right)=\left(\begin{array}{cc}a z F_{n+1}+a^{2} F_{n} & F_{n+1} \\ a z \cdot a^{2} F_{n}+a^{2} a^{2} F_{n-1} & a^{2} F_{n}\end{array}\right)=\left(\begin{array}{cc}F_{n+2} & F_{n+1} \\ a^{2} F_{n+1} & a^{2} F_{n}\end{array}\right)$, para todo $n \geq 1$, assim, ao calcularmos o determinante dessa matriz, teremos que: $\operatorname{det} A=-a^{2}$. Diante disso, em seguida, discutiremos a generalização de algumas identidades clássicas, que têm se tornado objeto de interesse em vários trabalhos.

\section{Propriedades e algumas identidades generalizadas}

Neste tópico, iremos explorar algumas identidades generalizadas, tais como de Cassini, d'Ocagne, Catalan e a fórmula de Honsberger, considerando-as nas variáveis complexas $a$ e $z$. Agora, a partir das matrizes do tipo $A^{n}=\left(\begin{array}{cc}F_{n+1} & F_{n} \\ a^{2} F_{n} & a^{2} F_{n-1}\end{array}\right)$, enunciamos os teoremas:

Teorema 4. ([16]) Identidade generalizada de Cassini: para $n \geq 1$, vale:

$$
F_{n+1}(a, z) F_{n-1}(a, z)-F_{n}(a, z)^{2}=(-1)^{n} a^{2 n-2} .
$$

Demonstração: basta ver o comportamento do seguinte determinante da matriz $\operatorname{det} A^{n}=\operatorname{det}\left(\begin{array}{cc}F_{n+1} & F_{n} \\ a^{2} F_{n} & a^{2} F_{n-1}\end{array}\right)$ e observamos que $\operatorname{det} A^{n}=\operatorname{det}(A \cdot \ldots \cdot A)=\operatorname{det}(A) \cdot \ldots \cdot \operatorname{det}(A)=\left(-a^{2}\right) \cdot \ldots \cdot\left(-a^{2}\right)=(-1)^{n} \cdot a^{2 n}$ Finalmente, ao lado direito, determinamos $\operatorname{det}\left(\begin{array}{cc}F_{n+1} & F_{n} \\ a^{2} F_{n} & a^{2} F_{n-1}\end{array}\right)=F_{n+1} \cdot a^{2} F_{n-1}-a^{2} F_{n} \cdot F_{n}=a^{2}\left(F_{n+1} F_{n-1}-F_{n}^{2}\right)$. Assim, segue o resultado pretendido.

Teorema 5. Para $m, n \geq 0$ temos que:

(i) $F_{n+m+1}(a, z)=F_{n+1}(a, z) F_{m+1}(a, z)+a^{2} \cdot F_{n}(a, z) F_{m}(a, z)$

(ii) $F_{n+m+p+1}(a, z)=F_{n+1}(a, z) F_{m+1}(a, z) F_{p+1}(a, z)+a^{2} \cdot F_{n}(a, z) F_{m}(a, z) F_{p+1}(a, z)+a^{2} \cdot F_{n+1}(a, z) F_{m}(a, z) F_{p}(a, z)$ $+a^{4} \cdot F_{n}(a, z) F_{m-1}(a, z) F_{p}(a, z)$.

Demonstração: (i) Vamos considerar a seguinte identidade matricial $A^{n+m}=A^{n} \cdot A^{m}$ e, a partir disso, observamos que $\left(\begin{array}{cc}F_{n+m+1} & F_{n+m} \\ a^{2} F_{n+m} & a^{2} F_{n+m-1}\end{array}\right)=A^{n+m}=\left(\begin{array}{cc}F_{n+1} & F_{n} \\ a^{2} F_{n} & a^{2} F_{n-1}\end{array}\right)\left(\begin{array}{cc}F_{m+1} & F_{m} \\ a^{2} F_{m} & a^{2} F_{m-1}\end{array}\right)$. Fazendo as contas, devemos determinar o produto matrizes $A^{n} \cdot A^{m}=\left(\begin{array}{cc}F_{n+1} F_{m+1}+F_{n} a^{2} F_{m} & F_{n+1} F_{m}+F_{n} a^{2} F_{m-1} \\ a^{2} F_{n} F_{m+1}+a^{4} F_{n-1} F_{m} & a^{2} F_{n} F_{m}+a^{4} F_{n-1} F_{m-1}\end{array}\right)$. De imediato, considerando o elemento da $1^{\mathrm{a}}$ linha e $1^{\mathrm{a}}$ coluna, determinaremos a identidade indicada no enunciado. Para determinar a identidade indicada no item (ii), vamos tomar $A^{n+m+p}=A^{n} \cdot A^{m} \cdot A^{p}$ e repetiremos os argumentos anteriores. 
Teorema 6. ([16]) Identidade generalizada de d'Ocagne: para os inteiros $m \geq n \geq 1$ temos que:

$$
F_{n+1}(a, z) \cdot F_{m}(a, z)-F_{n}(a, z) \cdot F_{m+1}(a, z)=(-1)^{n} a^{2 n} \cdot F_{m-n}(a, z) .
$$

Demonstração: para tanto, assumiremos a seguinte matriz $B_{0}=\left(\begin{array}{cc}F_{n+2} & F_{n+2} \\ a^{2} F_{n+1} & a^{2} F_{n+1}\end{array}\right)$ e, logo em seguida, para descrevermos a matriz $B_{1}$, vamos considerar a primeira coluna da soma da matriz $a^{2} A^{n}=\left(\begin{array}{cc}a^{2} F_{n+1} & a^{2} F_{n} \\ a^{4} F_{n} & a^{4} F_{n-1}\end{array}\right)$ com a seguinte matriz $a z \cdot B_{0}=\left(\begin{array}{cc}a z \cdot F_{n+2} & a z \cdot F_{n+2} \\ a^{3} z \cdot F_{n+1} & a^{3} z \cdot F_{n+1}\end{array}\right)$. Além disso, deixaremos fixa a segunda coluna da matriz $B_{0}$. Portanto, vamos tomar a seguinte expressão $B_{1}=\left(\begin{array}{cc}a z \cdot F_{n+2}+a^{2} F_{n+1} & F_{n+2} \\ a^{2}\left(a z \cdot F_{n+1}+a^{2} F_{n}\right) & a^{2} \cdot F_{n+1}\end{array}\right)=\left(\begin{array}{cc}F_{n+3} & F_{n+2} \\ a^{2} F_{n+2} & a^{2} F_{n+1}\end{array}\right)$. No passo seguinte, repetimos o processo, ao considerar a soma da primeira coluna da matriz $a^{2} A^{n+1}=\left(\begin{array}{cc}a^{2} F_{n+2} & a^{2} F_{n+1} \\ a^{4} F_{n+1} & a^{4} F_{n}\end{array}\right)$ com a primeira coluna da matriz que indicamos $a z \cdot B_{1}=\left(\begin{array}{cc}a z \cdot F_{n+3} & a z \cdot F_{n+2} \\ a^{3} z \cdot F_{n+2} & a^{3} z \cdot F_{n+1}\end{array}\right)$. Assim, determinaremos $B_{2}=\left(\begin{array}{cc}F_{n+4} & F_{n+2} \\ a^{2} F_{n+3} & a^{2} F_{n+1}\end{array}\right) \cdot$ Sucessivamente, determinaremos $B_{3}=\left(\begin{array}{cc}F_{n+5} & F_{n+2} \\ a^{2} F_{n+4} & a^{2} F_{n+1}\end{array}\right), B_{4}=\left(\begin{array}{cc}F_{n+6} & F_{n+2} \\ a^{2} F_{n+5} & a^{2} F_{n+1}\end{array}\right)$, $B_{5}=\left(\begin{array}{cc}F_{n+7} & F_{n+2} \\ a^{2} F_{n+6} & a^{2} F_{n+1}\end{array}\right), B_{6}=\left(\begin{array}{cc}F_{n+8} & F_{n+2} \\ a^{2} F_{n+7} & a^{2} F_{n+1}\end{array}\right), B_{7}=\left(\begin{array}{cc}F_{n+9} & F_{n+2} \\ a^{2} F_{n+8} & a^{2} F_{n+1}\end{array}\right), B_{8}=\left(\begin{array}{cc}F_{n+10} & F_{n+2} \\ a^{2} F_{n+9} & a^{2} F_{n+1}\end{array}\right), \ldots$, $B_{r}=\left(\begin{array}{cc}F_{n+r+2} & F_{n+2} \\ a^{2} F_{n+r+1} & a^{2} F_{n+1}\end{array}\right)$. A fim de verificarmos a matriz $\mathrm{B}_{\mathrm{r}}$, apresentaremos o seguinte lema:

Lema 2. Para $r \geq 0$ temos que:

$$
B_{r}=\left(\begin{array}{cc}
F_{n+r+2}(a, z) & F_{n+2}(a, z) \\
a^{2} F_{n+r+1}(a, z) & a^{2} F_{n+1}(a, z)
\end{array}\right)
$$

Demonstração: de imediato, vemos para $r=0$ e que $B_{0}=\left(\begin{array}{cc}F_{n+0+2} & F_{n+2} \\ a^{2} F_{n+0+1} & a^{2} F_{n+1}\end{array}\right)$ e para $r=1$ e que $B_{1}=\left(\begin{array}{cc}F_{n+3} & F_{n+2} \\ a^{2} F_{n+2} & a^{2} F_{n+1}\end{array}\right)=\left(\begin{array}{cc}F_{n+1+2} & F_{n+2} \\ a^{2} F_{n+1+1} & a^{2} F_{n+1}\end{array}\right)$. Por indução, investigaremos o comportamento da matriz $B_{r+1}=\left(\begin{array}{cc}? & F_{n+2} \\ ? & a^{2} F_{n+1}\end{array}\right)$, tendo em vista que a segunda coluna permanece fixa. Repetimos o mesmo argumento anterior, multiplicando as matrizes $a^{2} A^{n+r}=\left(\begin{array}{cc}a^{2} F_{n+r+1} & a^{2} F_{n+r} \\ a^{4} F_{n+r} & a^{4} F_{n+r-1}\end{array}\right)$ e 
$a z \cdot B_{r}=\left(\begin{array}{cc}a z \cdot F_{n+r+2} & a z \cdot F_{n+2} \\ a z \cdot a^{2} F_{n+r+1} & a z \cdot a^{2} F_{n+1}\end{array}\right)$. Dessa forma, tomaremos $B_{r+1}=\left(\begin{array}{cc}a^{2} F_{n+r+1}+a z \cdot F_{n+r+2} & F_{n+2} \\ a^{4} F_{n+r}+a z \cdot a^{2} F_{n+r+1} & a^{2} F_{n+1}\end{array}\right)=$ $=\left(\begin{array}{cc}F_{n+r+3} & F_{n+2} \\ a^{2}\left(a^{2} F_{n+r}+a z \cdot F_{n+r+1}\right) & a^{2} F_{n+1}\end{array}\right)=\left(\begin{array}{cc}F_{n+r+3} & F_{n+2} \\ a^{2} F_{n+r+2} & a^{2} F_{n+1}\end{array}\right)$, para todo $r \geq 0$.

A partir dessas relações, retornaremos para a demonstração do teorema 6. Assim, vamos notar que $\operatorname{det} B_{0}=\operatorname{det}\left(\begin{array}{cc}F_{n+2} & F_{n+2} \\ a^{2} F_{n+1} & a^{2} F_{n+1}\end{array}\right)=F_{n+2} a^{2} F_{n+1}-a^{2} F_{n+1} F_{n+2}=0$. E, a partir do teorema 4, devemos obter que $\operatorname{det} B_{1}=\operatorname{det}\left(\begin{array}{cc}F_{n+3} & F_{n+2} \\ a^{2} F_{n+2} & a^{2} F_{n+1}\end{array}\right)=a^{2}\left(F_{n+3} F_{n+1}-F_{n+2}{ }^{2}\right)=a^{2} \cdot(-1)^{n+2} \cdot a^{2 n+2}=(-1)^{n+2} \cdot a^{2 n+4} F_{1}$. Ou ainda que $\operatorname{det} B_{2}=\operatorname{det}\left(\begin{array}{cc}F_{n+4} & F_{n+2} \\ a^{2} F_{n+3} & a^{2} F_{n+1}\end{array}\right)=a^{2} \cdot\left(F_{n+4} \cdot F_{n+1}-F_{n+3} \cdot F_{n+2}\right)=a^{2}\left[\left(F_{n+1}\left(a \mathrm{z} F_{n+3}+a^{2} F_{n+2}\right)\right.\right.$ $\left.-F_{n+2}\left(a \mathrm{z} F_{n+2}+a^{2} F_{n+1}\right)\right]=a^{2}\left[F_{n+1} a \mathrm{z} F_{n+3}-F_{n+2} \cdot a \mathrm{z} F_{n+2}\right]=a^{2} \cdot a \mathrm{z} \cdot\left(F_{n+1} F_{n+3}-F_{n+2}^{2}\right)=a^{2} \cdot a \mathrm{z} \cdot\left[(-1)^{n+2} \cdot a^{2 n+2}\right]=$ $=(-1)^{n+2} \cdot a^{2 n+4} \cdot F_{2}$. Finalmente, pelo teorema 4 anteriormente discutido, determinamos $\operatorname{det} B_{2}$.

Vejamos mais um caso particular, na avaliação do determinante de $B_{3}=\left(\begin{array}{cc}F_{n+5} & F_{n+2} \\ a^{2} F_{n+4} & a^{2} F_{n+1}\end{array}\right)$. Nesse caso, temos $\operatorname{det} B_{3}=\operatorname{det}\left(\begin{array}{cc}F_{n+5} & F_{n+2} \\ a^{2} F_{n+4} & a^{2} F_{n+1}\end{array}\right)=a^{2}\left(F_{n+5} F_{n+1}-F_{n+4} F_{n+2}\right)$. Mais uma vez, fazendo pormenorizadamente as contas, veremos o comportamento de $\operatorname{det} B_{3}=a^{2}\left[\left(a \mathrm{z} F_{n+4}+a^{2} F_{n+3}\right) \cdot F_{n+1}\right.$ $\left.-\left(a z F_{n+3}+a^{2} F_{n+2}\right) \cdot F_{n+2}\right]=a^{2}\left[a z\left(\left(a z F_{n+3} F_{n+1}+a^{2} F_{n+2} F_{n+1}\right)+a^{2} F_{n+3} F_{n+1}-a z F_{n+3} F_{n+2}-a^{2} F_{n+2} F_{n+2}\right]=\right.$ $=a^{2}\left(\left(a^{2} z^{2}+a^{2}\right) F_{n+1} F_{n+3}-\left(a^{2} z^{2}+a^{2}\right) F_{n+2}^{2}\right)=a^{2}\left(F_{n+1} F_{n+3}-F_{n+2}^{2}\right)\left(a^{2} z^{2}+a^{2}\right)=a^{2}\left((-1)^{n+2} a^{2 n+2}\right) F_{3}=(-1)^{n+2} a^{2 n+4} \cdot F_{3}$.

De modo análogo, obtemos $\operatorname{det}_{4}, \operatorname{det} B_{5}, \operatorname{det} B_{6}, \ldots$, permitindo pensar em $\operatorname{det}_{r}=(-1)^{n+2} a^{2 n+4} \cdot F_{r}(a, z)$. Assim, assumiremos $\operatorname{det} B_{r}=\operatorname{det}\left(\begin{array}{cc}F_{n+r+2} & F_{n+2} \\ a^{2} F_{n+r+1} & a^{2} F_{n+1}\end{array}\right)=(-1)^{n+2} a^{2 n+4} \cdot F_{r}$. E, vendo que $\quad \operatorname{det} B_{r+1}=\operatorname{det}\left(\begin{array}{cc}F_{n+r+3} & F_{n+2} \\ a^{2} F_{n+r+2} & a^{2} F_{n+1}\end{array}\right)=\operatorname{det}\left(\begin{array}{cc}a z F_{n+r+2}+a^{2} F_{n+r+1} & F_{n+2} \\ a^{2}\left(a z F_{n+r+1}+a^{2} F_{n+r}\right) & a^{2} F_{n+1}\end{array}\right)=\operatorname{az} \cdot\left(\operatorname{det}\left(\begin{array}{cc}F_{n+r+2} & F_{n+2} \\ a^{2} F_{n+r+1} & a^{2} F_{n+1}\end{array}\right)\right)+$ $+a^{2} \cdot\left(\operatorname{det}\left(\begin{array}{cc}F_{n+r+1} & F_{n+2} \\ a^{2} F_{n+r} & a^{2} F_{n+1}\end{array}\right)\right)=a z \cdot\left(F_{n+r+2} a^{2} F_{n+1}-a^{2} F_{n+r+1} \cdot F_{n+2}\right)+a^{2}\left(F_{n+r+1} a^{2} F_{n+1}-a^{2} F_{n+r} F_{n+2}\right)=$ $=a z \cdot\left[(-1)^{n+2} a^{2 n+4} \cdot F_{r}\right]+a^{2}\left[(-1)^{n+2} a^{2 n+4} \cdot F_{r-1}\right]=(-1)^{n+2} a^{2 n+4}\left(a z \cdot F_{r}+a^{2} F_{r-1}\right)$. Isto determinamos que det $B_{r+1}=(-1)^{n+2} a^{2 n+4}\left(a z \cdot F_{r}+a^{2} F_{r-1}\right)=(-1)^{n+2} a^{2 n+4} \cdot F_{r+1}$.

E, fazendo a seguinte substituição $m=n+r$ e como já sabemos que $\operatorname{det} B_{r}=a^{2}\left(F_{n+r+2} F_{n+1}-F_{n+r+1} F_{n+2}\right)=(-1)^{n+2} a^{2 n+4} \cdot F_{r}$, devemos determinar finalmente que vale $a^{2}\left(F_{m+2} F_{n+1}-F_{m+1} F_{n+2}\right)=(-1)^{n+2} a^{2 n+4} \cdot F_{m-n}$

Assim, com origem nos argumentos anteriores, segue a identidade de d'Ocagne. Vejamos a seguir, a generalização de outra identidade clássica. 
Teorema 7. ([16]) Identidade generalizada de Catalan: para os inteiros $\mathrm{m} \geq n \geq 1$ temos que:

$$
F_{n}(a, z) F_{m}(a, z)-F_{n-r}(a, z) F_{m+r}(a, z)=(-1)^{n-r} a^{2 n-2 r} \cdot F_{m-n+r}(a, z) \cdot F_{r}(a, z) .
$$

Demonstração: definiremos a seguinte matriz $C_{0}=\left(\begin{array}{cc}F_{n} & F_{n-r} \\ a^{2} F_{n+1} & a^{2} F_{n+1}\end{array}\right)$. Agora, consideremos a matriz $B_{r}=\left(\begin{array}{cc}F_{n+r+2} & F_{n+2} \\ a^{2} F_{n+r+1} & a^{2} F_{n+1}\end{array}\right)$ e, em seguida, substituiremos o índice ' $n$ ' por ' $n-r-1$ ' na mesma matriz $\quad B_{r} \quad$ e obteremos $\left(\begin{array}{cc}F_{n-r-1+r+2} & F_{n-r-1+2} \\ a^{2} F_{n-r-1+r+1} & a^{2} F_{n-r-1+1}\end{array}\right)=\left(\begin{array}{cc}F_{n+1} & F_{n-r+1} \\ a^{2} F_{n} & a^{2} F_{n-r}\end{array}\right)$. Doravante, designaremos $C_{1}=\left(\begin{array}{cc}F_{n+1} & F_{n-r+1} \\ a^{2} F_{n} & a^{2} F_{n-r}\end{array}\right)$. Em seguida, construiremos a matriz $C_{2}$, tomando a primeira linha da matriz há pouco definida por $C_{0}$ multiplicada pelo termo $\left(a^{2}\right)$, para a linha correspondente da outra matriz indicada por $C_{0}$, e adicionando a primeira linha da matriz $C_{1}$, quando multiplicada por (az). Por tal regra, determinaremos a matriz $C_{2}=\left(\begin{array}{cc}a^{2} F_{n}+a z \cdot F_{n+1} & a^{2} F_{n-r}+a \mathrm{Z} \cdot F_{n-r+1} \\ a^{2} F_{n} & a^{2} F_{n-r}\end{array}\right)=\left(\begin{array}{cc}F_{n+2} & F_{n-r+2} \\ a^{2} F_{n} & a^{2} F_{n-r}\end{array}\right) . \quad$ Recorrendo à definição 2, observamos que $F_{n+2}=a^{2} F_{n}+a z \cdot F_{n+1}$ e que também $F_{n-r+2}=a^{2} F_{n-r}+a \mathrm{z} \cdot F_{n-r+1}$.

No passo seguinte, a fim de determinar a matriz $C_{3}$, tomaremos de novo a primeira linha da matriz $C_{1}$ multiplicada por $\left(a^{2}\right)$ e adicionando a primeira linha da matriz $C_{2}$ multiplicada pelo termo $(a z)$. Observe que deixamos fixa a segunda linha, para escrever $C_{3}=\left(\begin{array}{cc}a^{2} F_{n+1}+\mathrm{az} \cdot F_{n+2} & a^{2} \cdot F_{n-r+1}+a z \cdot F_{n-r+2} \\ a^{2} F_{n} & a^{2} F_{n-r}\end{array}\right) . \quad$ E, reparando que $a^{2} F_{n+1}+a z \cdot F_{n+2}=F_{n+3} \quad$ e que $a^{2} \cdot F_{n-r+1}+a z \cdot F_{n-r+2}=F_{n-r+3}$ podemos determinar $C_{3}=\left(\begin{array}{cc}F_{n+3} & F_{n-r+3} \\ a^{2} F_{n} & a^{2} F_{n-r}\end{array}\right)$.

Com o escopo de confirmar nosso raciocínio, vamos descrever a matriz da ordem subsequente, que indicaremos por $C_{4}=\left(\begin{array}{cc}? & ? \\ a^{2} F_{n} & a^{2} F_{n-r}\end{array}\right)$ e, a fim de determinarmos sua primeira linha, tomaremos a primeira linha da matriz $C_{2}$ multiplicada por $\left(a^{2}\right)$ e adicionando a primeira linha da matriz $C_{3}$ multiplicada pelo termo $(a z)$. Daí, obteremos $C_{4}=\left(\begin{array}{cc}a^{2} F_{n+2}+a z \cdot F_{n+3} & a^{2} F_{n-r+2}+a z \cdot F_{n-r+3} \\ a^{2} F_{n} & a^{2} F_{n-r}\end{array}\right)=\left(\begin{array}{cc}F_{n+4} & F_{n-r+4} \\ a^{2} F_{n} & a^{2} F_{n-r}\end{array}\right)$.

Antes de prosseguirmos na demonstração, vejamos o lema 3, que é uma representação generalizada dessas construções matriciais de $C_{s}$.

Lema 3. Para $s \geq 2$, temos que: 


$$
C_{s}=\left(\begin{array}{cc}
F_{n+s}(a, z) & F_{n-r+s}(a, z) \\
a^{2} F_{n}(a, z) & a^{2} F_{n-r}(a, z)
\end{array}\right)
$$

Demonstração: vimos anteriormente, alguns casos particulares e procedendo por indução, vejamos que a matriz $C_{s+1}=\left(\begin{array}{cc}? & ? \\ a^{2} F_{n} & a^{2} F_{n-r}\end{array}\right)$ deve ser determinada, partindo da primeira linha da matriz $C_{s-1}=\left(\begin{array}{cc}F_{n+s-1} & F_{n-r+s-1} \\ a^{2} F_{n} & a^{2} F_{n-r}\end{array}\right)$ multiplicada por $\left(a^{2}\right)$ e adicionando a primeira linha da matriz $C_{s}=\left(\begin{array}{cc}F_{n+s} & F_{n-r+s} \\ a^{2} F_{n} & a^{2} F_{n-r}\end{array}\right) \quad$ multiplicada pelo termo $(a z)$. Dessa forma, teremos $C_{s+1}=\left(\begin{array}{cc}a^{2} F_{n+s-1}+a z \cdot F_{n+s} & a^{2} F_{n-r+s-1}+a z \cdot F_{n-r+s} \\ a^{2} F_{n} & a^{2} F_{n-r}\end{array}\right)=\left(\begin{array}{cc}F_{n+s+1} & F_{n-r+s+1} \\ a^{2} F_{n} & a^{2} F_{n-r}\end{array}\right)$. Assim, segue o resultado que pretendíamos.

Além do mais, podemos escrever que $\operatorname{det}\left(\begin{array}{cc}F_{n+s} & F_{n-r+s} \\ a^{2} F_{n} & a^{2} F_{n-r}\end{array}\right)=\operatorname{det} C_{s}=a z \cdot \operatorname{det} C_{s-1}+a^{2} \operatorname{det} C_{s-2}=$ $=a z \cdot \operatorname{det}\left(\begin{array}{cc}F_{n+s-1} & F_{n-r+s-1} \\ a^{2} F_{n} & a^{2} F_{n-r}\end{array}\right)+a^{2} \cdot \operatorname{det}\left(\begin{array}{cc}F_{n+s-2} & F_{n-r+s-2} \\ a^{2} F_{n} & a^{2} F_{n-r}\end{array}\right)$. Logo em seguida, apresentaremos o lema 4, que permite a descrição do comportamento dos determinantes das matrizes que indicamos por $C_{0}, C_{1}, C_{2}, C_{3}, C_{4}, \ldots, C_{s}$.

Lema 4. Para $s \geq 2$, temos que:

$$
\operatorname{det} C_{s}=\operatorname{det}\left(\begin{array}{cc}
F_{n+s}(a, z) & F_{n-r+s}(a, z) \\
a^{2} F_{n}(a, z) & a^{2} F_{n-r}(a, z)
\end{array}\right)=(-1)^{n-r+1} a^{2 n-2 r+2} F_{r}(a, z) F_{s}(a, z)
$$

Demonstração: preliminarmente, temos que $\operatorname{det} C_{0}=a^{2} F_{n} F_{n+1}-a^{2} F_{n-r} F_{n+1}$. Ademais, recordamos que a partir da matriz $B_{r}$, cujo determinante é obtido por $\operatorname{det} B_{r}=(-1)^{n+2} a^{2 n+4} \cdot F_{r}$, nesse sentido, procedemos uma substituição imediata do índice ' $n$ ' pelo índice ' $n-r-1$ ', assim, devemos encontrar que $\operatorname{det} B_{r}=\operatorname{det} C_{1}=(-1)^{(n-r-1)+2} a^{2(n-r-1)+4} \cdot F_{r}=(-1)^{n-r+1} a^{2 n-2 r+2} \cdot F_{r}$. Por outro lado, sabemos ainda que $\operatorname{det} C_{1}=a^{2}\left(F_{n+1} F_{n-r}-F_{n-r+1} F_{n}\right)$, que implica na seguinte igualdade $a^{2}\left(F_{n+1} F_{n-r}-F_{n-r+1} F_{n}\right)=(-1)^{n-r+1} a^{2 n-2 r+2} \cdot F_{r}$, portanto, vem que $\operatorname{det} C_{1}=\left(F_{n+1} F_{n-r}-F_{n-r+1} F_{n}\right)=$ $=(-1)^{n-r+1} a^{2 n-2 r+2} \cdot F_{r} \cdot F_{1}$.

Por conseguinte, por indução sobre ' $s$ ' e posto que recorremos à seguinte identidade envolvendo determinantes: $\operatorname{det} C_{s}=a z \cdot \operatorname{det} C_{s-1}+a^{2} \operatorname{det} C_{s-2}$. Com isso, podemos descrever $\operatorname{det} C_{2}=a z \cdot \operatorname{det} C_{1}+a^{2} \operatorname{det} C_{0}=a z \cdot(-1)^{n-r+1} a^{2 n-2 r+2} \cdot F_{r}+0=(-1)^{n-r+1} a^{2 n-2 r+2} \cdot F_{r} \cdot F_{2}$, em seguida, usaremos o mesmo raciocínio para $\operatorname{det} C_{3}=a z \cdot \operatorname{det} C_{2}+a^{2} \operatorname{det} C_{1}=a z \cdot\left[(-1)^{n-r+1} \cdot a^{2 n-2 r+2} \cdot F_{r} \cdot F_{2}\right]+a^{2}\left[(-1)^{n-r+1} \cdot a^{2 n-2 r+2} \cdot F_{r} \cdot F_{1}\right]=$ $=(-1)^{n-r+1} \cdot a^{2 n-2 r+2} \cdot F_{r}\left(a z \cdot F_{2}+a^{2} \cdot F_{1}\right)=(-1)^{n-r+1} \cdot a^{2 n-2 r+2} \cdot F_{r} \cdot F_{3} \cdot$ A fim de comprovar nossa argumentação e descrever nossa hipótese de indução, vejamos ainda o próximo caso que 
indicamos

$\operatorname{det}_{4}=a z \cdot \operatorname{det} C_{3}+a^{2} \operatorname{det} C_{2}=a z \cdot\left[(-1)^{n-r+1} \cdot a^{2 n-2 r+2} \cdot F_{r} \cdot F_{3}\right]+a^{2}\left[(-1)^{n-r+1} \cdot a^{2 n-2 r+2} \cdot F_{r} \cdot F_{2}\right]=$ $=(-1)^{n-r+1} \cdot a^{2 n-2 r+2} \cdot F_{r} \cdot\left(a z \cdot F_{3}+a^{2} \cdot F_{2}\right)=(-1)^{n-r+1} \cdot a^{2 n-2 r+2} \cdot F_{r} \cdot F_{4}$.

Para concluir, por indução e empregando a identidade $\operatorname{det} C_{s}=a z \cdot \operatorname{det} C_{s-1}+a^{2} \operatorname{det} C_{s-2}$, segue que $\quad \operatorname{det} C_{s}=a z \cdot \operatorname{det} C_{s-1}+a^{2} \operatorname{det} C_{s-2}=a z\left[(-1)^{n-r+1} \cdot a^{2 n-2 r+2} \cdot F_{r} \cdot F_{s-1}\right]+a^{2}\left[(-1)^{n-r+1} \cdot a^{2 n-2 r+2} \cdot F_{r} \cdot F_{s-2}\right]=$ $=(-1)^{n-r+1} a^{2 n-2 r+2} \cdot F_{r} \cdot\left(a z \cdot F_{s-1}+a^{2} \cdot F_{s-2}\right)=(-1)^{n-r+1} a^{2 n-2 r+2} \cdot F_{r} \cdot F_{s}$, para todo $s \geq 2$.

Retomando a demonstração do teorema 7, seguimos avaliando a seguinte expressão $\operatorname{det} C_{s}=\operatorname{det}\left(\begin{array}{cc}F_{n+s} & F_{n-r+s} \\ a^{2} F_{n} & a^{2} F_{n-r}\end{array}\right)=a^{2}\left(F_{n-r} F_{n+s}-F_{n-r+s} F_{n}\right)$ e realizando a substituição $s=m-n+r$, assim, podemos escrever a expressão $a^{2}\left(F_{n-r} F_{n+m-n+r}-F_{n-r+m-n+r} F_{n}\right)=a^{2}\left(F_{n-r} F_{m+r}-F_{m} F_{n}\right)$. Finalmente, tendo em vista a obtenção da fórmula generalizada de Catalan, vamos empregar a identidade. Dessa forma, encontramos a igualdade indicada por $a^{2}\left(F_{n-r} F_{m+r}-F_{m} F_{n}\right)=(-1)^{n-r+1} a^{2 n-2 r+2} \cdot F_{r} \cdot F_{s}$ para $m \geq n \geq 1$ e $r, s \geq 0$, simplificando teremos $\left(F_{m} F_{n}-F_{n-r} F_{m+r}\right)=(-1)^{n-r} \cdot a^{2 n-2 r} \cdot F_{r} \cdot F_{m-n+r}$.

É relevante observar o comportamento de casos particulares. Com efeito, na identidade generalizada de Catalan, quando tomamos $m=n$, encontraremos a identidade de Catalan conhecida por $F_{n}^{2}-F_{n-r} F_{m+r}=(-1)^{n-r} a^{2 n-2 r} \cdot F_{m-n+r} \cdot F_{r}=(-1)^{n-r} a^{2 n-2 r} \cdot F_{r}^{2}$. Ademais, quando tomamos $r=1$, obtemos $F_{n}^{2}-F_{n-1} F_{n+1}=(-1)^{n-1} a^{2 n-2} \cdot F_{1}^{2}=(-1)^{n-1} a^{2 n-2}$, o que determina a identidade de Cassini.

Antes de finalizarmos a presente seção, a partir das representações matriciais discutidas, podem ser obtidas uma série de propriedades para índices inteiros. Por exemplo, desde que $A^{n}=\left(\begin{array}{cc}F_{n+1} & F_{n} \\ a^{2} F_{n} & a^{2} F_{n-1}\end{array}\right)$, para todo $n \geq 0$, com o emprego do teorema 3, escrevemos $A^{-n}=\left(\begin{array}{cc}F_{-n+1} & F_{-n} \\ a^{2} F_{-n} & a^{2} F_{-n-1}\end{array}\right)=$ $=\left(\begin{array}{cc}F_{-(n-1)} & F_{-n} \\ a^{2} F_{-n} & a^{2} F_{-(n+1)}\end{array}\right)=\left(\begin{array}{cc}\frac{1}{(-1)^{n-1+1} a^{2(n-1)}} F_{n-1} & \frac{1}{(-1)^{n+1} a^{2 n}} F_{n} \\ a^{2} \frac{1}{(-1)^{n+1} a^{2 n}} F_{n} & a^{2} \frac{1}{(-1)^{n+1+1} a^{2(n+1)}} F_{n+1}\end{array}\right)=\left(\begin{array}{cc}\frac{-1}{(-1)^{n+1} a^{2 n-2}} F_{n-1} & \frac{1}{(-1)^{n+1} a^{2 n}} F_{n} \\ a^{2} \frac{1}{(-1)^{n+1} a^{2 n}} F_{n} & a^{2} \frac{-1}{(-1)^{n+1} a^{2 n+2}} F_{n+1}\end{array}\right)=$ $=\left(\begin{array}{cc}\frac{-1}{(-1)^{n+1} a^{2 n-2}} F_{n-1} & \frac{1}{(-1)^{n+1} a^{2 n}} F_{n} \\ a^{2} \frac{1}{(-1)^{n+1} a^{2 n}} F_{n} & a^{2} \frac{-1}{(-1)^{n+1} a^{2 n+2}} F_{n+1}\end{array}\right)=\frac{1}{(-1)^{n+1} a^{2 n}}\left(\begin{array}{cc}-a^{2} F_{n-1} & F_{n} \\ a^{2} F_{n} & -F_{n+1}\end{array}\right)$. Portanto, temos a seguinte identidade $A^{-n}=\frac{1}{(-1)^{n+1} a^{2 n}} A^{*}$, onde $A^{*}=\left(\begin{array}{cc}-a^{2} F_{n-1} & F_{n} \\ a^{2} F_{n} & -F_{n+1}\end{array}\right)$. ̀̀ vista disso, a seguir, as identidades serão discutidas para índices inteiros.

\section{Extensão das identidades para índices inteiros.}

Na seção passada estudamos uma série de identidades famosas que mantiveram a atenção de vários matemáticos, desde os séculos passados. Agora, a partir dos resultados indicados em [16], conhecemos a identidade de Cassini, que foi descrita no teorema 4. Ademais, deduzimos 
ainda as identidades de d'Ocagne e a forma generalizada de Catalan, respectivamente, nos teoremas 6 e 7. Por fim, apresentamos a fórmula (generalizada) de Honsberger [16]:

$$
F_{n+m+1}(a, z)=F_{n+1}(a, z) F(a, z)_{m+1}+a^{2} \cdot F_{n}(a, z) \cdot F_{m}(a, z), \text { para } n, m \geq 0 .
$$

Todavia, todas foram deduzidas ao se levar em consideração índices inteiros positivos. Doravante, aplicando o teorema 3, vamos investigar o comportamento das identidades clássicas de Cassini, d’Ocagne, Catalan e a fórmula de Honsberger, assim considerando índices inteiros quaisquer. No nosso primeiro caso, temos $F_{-n+1} F_{-n-1}-F_{-n}{ }^{2}=F_{-(n-1)} F_{-(n+1)}-F_{-n}{ }^{2}=$ $=\frac{1}{(-1)^{(n-1)+1} a^{2(n-1)}} F_{n-1} \cdot \frac{1}{(-1)^{(n+1)+1} a^{2(n+1)}} F_{n+1}-\left[\frac{1}{(-1)^{n+1} a^{2 n}} F_{n}\right]^{2}=\frac{1}{(-1)^{n} a^{2 n-2}} F_{n-1} \cdot \frac{1}{(-1)^{n+2} a^{2 n+2}} F_{n+1}$ $-\frac{1}{(-1)^{2 n+2} a^{4 n}} F_{n}{ }^{2}=\frac{1}{(-1)^{2 n+2} a^{4 n}} F_{n-1} F_{n+1}-\frac{1}{(-1)^{2 n+2} a^{4 n}} F_{n}{ }^{2}=\frac{1}{(-1)^{2 n+2} a^{4 n}}\left(F_{n-1} F_{n+1}-F_{n}{ }^{2}\right)=$ $=\frac{1}{(-1)^{2 n+2} a^{4 n}} \cdot\left((-1)^{n} a^{2 n-2}\right)=\frac{1}{(-1)^{n+2} a^{2 n+2}}$. Isto é, para ' $n$ ' inteiro qualquer, a fórmula de Cassini pode ser expressa por:

$$
F_{-n+1}(a, z) F_{-n-1}(a, z)-F_{-n}(a, z)^{2}=(-1)^{-n} a^{-2 n-2} .
$$

No segundo caso, investigaremos a identidade de d'Ocagne, determinada como $F_{n+1} F_{m}-F_{n} F_{m+1}=(-1)^{n} a^{2 n} \cdot F_{m-n}$, para $n \geq m \geq 1$. Assim, consideraremos $F_{-n+1} F_{-m}-F_{-n} F_{-m+1}=$ $=F_{-(n-1)} F_{-m}-F_{-n} F_{-(m-1)}==\frac{1}{(-1)^{n} a^{2 n-2}} F_{n-1} \frac{1}{(-1)^{m+1} a^{2 m}} F_{m}-\frac{1}{(-1)^{n+1} a^{2 n}} F_{n} \frac{1}{(-1)^{m} a^{2 m-2}} F_{m-1}=\frac{1}{(-1)^{n+m+1} a^{2 n+2 m-2}}\left(F_{n-1} F_{m}-F_{n} F_{m-1}\right)=$ $=\frac{1}{(-1)^{n+m} a^{2 n+2 m-2}}\left(F_{n} F_{m-1}-F_{n-1} F_{m}\right)=\frac{1}{(-1)^{n+m} a^{2 n+2 m-2}}\left[(-1)^{n-1} a^{2(n-1)} \cdot F_{(m-1)-(n-1)}\right]=(-1)^{-m-1} a^{-2 m} F_{m-n}$. Com isso, para ' $m$ ' e ' $n$ ' inteiros quaisquer, vemos que:

$$
F_{-n+1}(a, z) F_{-m}(a, z)-F_{-n}(a, z) F_{-m+1}(a, z)=(-1)^{-m-1} a^{-2 m} F_{m-n}(a, \mathrm{z}) .
$$

Para a identidade de Catalan, vimos que $F_{n} F_{m}-F_{n-r} F_{m+r}=(-1)^{n-r} a^{2 n-2 r} \cdot F_{m-n+r} \cdot F_{r}$, para $m \geq n \geq 1$ e todo $r \geq 0$. Assim, vejamos o que ocorre com a substituição imediata no teorema 3 : $F_{-n} \cdot F_{-m}-F_{-n-r} \cdot F_{-m+r}=F_{-n} \cdot F_{-m}-F_{-(n+r)} \cdot F_{-(m-r)}=\frac{1}{(-1)^{n+1} a^{2 n}} F_{n} \frac{1}{(-1)^{m+1} a^{2 m}} F_{m}-\frac{1}{(-1)^{n+r+1} a^{2 n+2 r}} F_{n+r} \frac{1}{(-1)^{m-r+1} a^{2 m-2 r}} F_{m-r}=$ $=\frac{1}{(-1)^{n+m+2} a^{2 n+2 m}} F_{n} F_{m}-\frac{1}{(-1)^{n+m+2} a^{2 n+2 m}} F_{n+r} F_{m-r}=\frac{1}{(-1)^{n+m+2} a^{2 n+2 m}}\left(F_{n} F_{m}-F_{n+r} F_{m-r}\right)$. Ou ainda, para inteiros quaisquer, determinamos que $F_{-n} \cdot F_{-m}-F_{-n-r} \cdot F_{-m+r}=\frac{1}{(-1)^{n+m+2} a^{2 n+2 m}}(-1)^{n+r} a^{2 n+2 r} \cdot F_{m-n-r} \cdot F_{-r}$ e, efetuando algumas simplificações, obteremos:

$$
F_{-n}(a, z) \cdot F_{-m}(a, z)-F_{-n-r}(a, z) \cdot F_{-m+r}(a, z)=(-1)^{-m+r} a^{-2 m+2 r} \cdot F_{m-n-r}(a, z) \cdot F_{-r}(a, z) .
$$


De modo análogo, para a fórmula de Honsberger encontraremos $F_{-n-m+1}=F_{-n+1} F_{-m+1}+a^{2} \cdot F_{-n} F_{-m}=F_{-(n-1)} F_{-(m-1)}+a^{2} \cdot F_{-n} F_{-m}=\frac{1}{(-1)^{n} a^{2 n-2}} F_{n-1} \frac{1}{(-1)^{m} a^{2 m-2}} F_{m-1}+a^{2} \cdot \frac{1}{(-1)^{n+1} a^{2 n}} F_{n} \frac{1}{(-1)^{m+1} a^{2 m}} F_{m}=$ $=\frac{1}{(-1)^{n+m} a^{2 n+2 m-2}}\left(a^{2} \cdot F_{n-1} \cdot F_{m-1}+F_{n} \cdot F_{m}\right)$. Por conseguinte, para quaisquer inteiros $m, n$, resulta na seguinte igualdade:

$$
F_{-n-m+1}(a, \mathrm{z})=F_{-n+1}(a, z) F_{-m+1}(a, z)+a^{2} \cdot F_{-n}(a, z) F_{-m}(a, z)=(-1)^{-n-m} \cdot a^{-2 n-2 m+2} \cdot F_{n+m-1}(a, \mathrm{z}) .
$$

A seguir, discutiremos as identidades para o caso particular na variável complexa $z$.

\section{Representação das identidades na variável $z$.}

Nesta seção, vamos explorar as identidades de Cassini, d’ocagne, Catalan e a fórmula de Honsberger, considerando-as, no caso particular, na variável complexa $z$. Desse modo, iremos empregar o lema 1. Com origem na identidade de Cassini, podemos determinar $F_{n+1} F_{n-1}-F_{n}^{2}=a^{n} \cdot f_{n+1}(z) a^{n-2} \cdot f_{n-1}(z)-a^{n-1} \cdot f_{n}(z) a^{n-1} \cdot f_{n}(z)=a^{2 n-2} \cdot\left(f_{n+1}(z) f_{n-1}(z)-f_{n}(z) f_{n}(z)\right)=$ $=(-1)^{n} a^{2 n-2}$, logo, para $m \geq n \geq 1$ encontramos:

$$
\left(f_{n+1}(z) f_{n-1}(z)-f_{n}(z) f_{n}(z)\right)=(-1)^{n} \text {. }
$$

Semelhantemente, para a identidade de d'Ocagne, devemos encontrar $F_{n+1} F_{m}-F_{n} F_{m+1}=$ $=\mathrm{a}^{n} \cdot f_{n+1}(z) \cdot \mathrm{a}^{m-1} \cdot f_{m}(z)-\mathrm{a}^{n-1} \cdot f_{n}(z) \cdot \mathrm{a}^{m} \cdot f_{m+1}(z)=\mathrm{a}^{n+m-1} \cdot\left(f_{n+1}(z) \cdot f_{m}(z)-f_{n}(z) \cdot f_{m+1}(z)\right)=(-1)^{n} a^{2 n} \cdot F_{m-n}$. Dessa última igualdade, para $m \geq n \geq 1$, determinamos que:

$$
f_{n+1}(z) \cdot f_{m}(z)-f_{n}(z) \cdot f_{m+1}(z)=(-1)^{n} \cdot a^{n-m+1} \cdot f_{m-n}(z)
$$

Nesse sentido, a identidade generalizada de Catalan nos possibilita considerar $F_{n} F_{m}-F_{n-r} F_{m+r}=a^{n-1} \cdot f_{n}(z) a^{m-1} \cdot f_{m}(z)-a^{n-r-1} \cdot f_{n-r}(z) a^{m+r-1} \cdot f_{m+r}(z)=a^{n+m-2} \cdot\left(f_{n}(z) \cdot f_{m}(z)-f_{n-r}(z) \cdot f_{m+r}(z)\right)=$ $=(-1)^{n-r} a^{2 n-2 r} \cdot F_{m-n+r} \cdot F_{r}$. E, para $m \geq n \geq 1$, efetuando alguns cancelamentos na última igualdade, encontramos:

$$
\left(f_{n}(z) \cdot f_{m}(z)-f_{n-r}(z) \cdot f_{m+r}(z)\right)=(-1)^{n-r} \cdot a^{n-m-2 r+2} \cdot f_{m-n+r}(z) \cdot f_{r}(z) .
$$

Finalmente, a fórmula de Honsberger $F_{n+m+1}=F_{n+1} F_{m+1}+a^{2} \cdot F_{n} F_{m}$ pode ser descrita, aplicando o lema 1 nos dois membros da igualdade: $a^{n+m} \cdot f_{n+m+1}(z)=a^{n} \cdot f_{n+1}(z) a^{m} \cdot f_{m+1}(z)+a^{2} \cdot a^{n-1} \cdot f_{n}(z) a^{m-1} \cdot f_{m}(z)$ ou, de modo simplificado, para $n, m \geq 0$ obtemos:

$$
f_{n+m+1}(z)=f_{n+1}(z) f_{m+1}(z)+f_{n}(z) \cdot f_{m}(z) \text {. }
$$

Por conseguinte, seguindo o processo de complexificação da sequência de Fibonacci, serão discutidos os quaternions complexos de Fibonacci.

\section{Quaternions complexos de Fibonacci de ordem ' $n$ '.}


$\mathrm{Na}$ última seção apresentamos uma nova definição, recordando a considerável tradição de investigações em torno dos quaternions complexos de Fibonacci, desde os anos 60 [10, 11, 14]. Para tanto, considerando a base canônica $\{1, i, j, k\}$, conhecida como a base usual do conjunto dos quaternions $q=q_{0}+q_{1} i+q_{2} j+q_{3} k$, cujos coeficientes são números reais. O referido conjunto é conhecido como uma Álgebra de dimensão quatro [10, 11].

Definição 4. Os Quaternions Complexos de Fibonacci de ordem ' $n$ ', nas variáveis $a$ e $z$, tem seus elementos definidos pela seguinte recorrência:

$$
Q_{n}(a, z)=F_{n}(a, z)+F_{n+1}(a, z) i+F_{n+2}(a, z) j+F_{n+3}(a, z) k, \text { para } n \geq 0 .
$$

De imediato, poderemos determinar alguns termos da sequência $\left\{Q_{n}(a, z)\right\}_{n \in I N}$ :

$$
\left\{\begin{array}{l}
Q_{0}=F_{0}+F_{1} i+F_{2} j+F_{3} k=0+i+a z \cdot j+\left(a^{2} z^{2}+a^{2}\right) \cdot k \\
Q_{1}=F_{1}+F_{2} i+F_{3} j+F_{4} k=1+a z \cdot i+\left(a^{2} z^{2}+a^{2}\right) \cdot j+\left(a^{3} z^{3}+2 a^{3} z\right) \cdot k \\
Q_{2}=F_{2}+F_{3} i+F_{4} j+F_{5} k=a z+\left(a^{2} z^{2}+a^{2}\right) \cdot i+\left(a^{3} z^{3}+2 a^{3} z\right) \cdot j+\left(a^{4} z^{4}+3 a^{4} z^{2}+a^{4}\right) \cdot k \\
\quad \vdots \\
\quad \vdots \\
Q_{n}=F_{n}+F_{n+1} i+F_{n+2} j+F_{n+3} k
\end{array}\right.
$$

Com isso, apresentamos o seguinte teorema:

Teorema 8. Para os Quaternions Complexos de Fibonacci de ordem $n$, nas variáveis $a$ e $z$, temos a relação de recorrência indicada por:

$$
Q_{n+1}(a, z)=a z \cdot Q_{n}(a, z)+a^{2} Q_{n-1}(a, z), \text { para todo inteiro } n \geq 0 \text {. }
$$

Demonstração: tendo em vista, agrupar os termos convenientes, relativamente à base canônica $\{1, i, j, k\}$. Podemos observar que ocorre $a z \cdot Q_{n}+a^{2} Q_{n-1}=a z\left(F_{n}+F_{n+1} i+F_{n+2} j+F_{n+3} k\right)+$ $+a^{2}\left(F_{n-1}+F_{n} i+F_{n+1} j+F_{n+2} k\right)=\left(a z F_{n}+a^{2} F_{n-1}\right)+\left(a \mathrm{z} F_{n+1}+a^{2} F_{n}\right) i+\left(a \mathrm{z} F_{n+2}+a^{2} F_{n+1}\right) j+$ $\left(a \mathrm{z} F_{n+3}+a^{2} F_{n+2}\right) k=F_{n+1}+F_{n+2} i+F_{n+3} j+F_{n+4} k=Q_{n+1}$, assim, verificamos a relação de recorrência enunciada neste teorema.

Além do mais, aplicaremos o teorema 3, a fim procedermos um processo de extensão para índices inteiros dos termos da sequência $\left\{Q_{n}\right\}_{n \in \mathbb{N}}$. Para isso, apresentaremos o corolário 1. Vejamos:

Corolário 1. Um Quaternion Complexo de Fibonacci de ordem ' $n$ ' pode ser descrito, nas variáveis $a$ e $z$,para índices negativos da seguinte forma:

$$
Q_{-n}(a, z)=(-1)^{-n-1} a^{-2 n}\left(F_{n}(a, \mathrm{z})-a^{2} F_{n-1}(a, \mathrm{z}) \cdot i+a^{4} F_{n-2}(a, \mathrm{z}) \cdot j-a^{6} F_{n-3}(a, \mathrm{z}) \cdot k\right) .
$$

Demonstração: partindo do teorema 3, podemos escrever $Q_{-n}=F_{-n}+F_{-n+1} i+F_{-n+2} j+$ 
$+F_{-n+3} k=F_{-n}+F_{-(n-1)} i+F_{-(n-2)} j+F_{-(n-3)} k=\frac{1}{(-1)^{n+1} a^{2 n}} \cdot F_{n}+\frac{1}{(-1)^{n} a^{2 n-2}} \cdot F_{n-1} \cdot i+$ $+\frac{1}{(-1)^{n-1} a^{2 n-4}} \cdot F_{n-2} \cdot j+\frac{1}{(-1)^{n-2} a^{2 n-6}} \cdot F_{n-3} \cdot k$. Portanto, colocando os termos comuns em evidencia, teremos que: $Q_{-n}=\frac{1}{(-1)^{n+1} a^{2 n}}\left(F_{n}-a^{2} F_{n-1} \cdot i+a^{4} F_{n-2} \cdot j-a^{6} F_{n-3} \cdot k\right)$.

Agora, recordando ao que foi discutido na seção 2.1, relativamente às propriedades da equação característica $t^{2}-a z \cdot t-a^{2}=0$ e diante do teorema 8 , compreendemos que a sequências de números $Q_{n}=F_{n}+F_{n+1} i+F_{n+2} j+F_{n+3} k$ e $\left\{F_{n}\right\}_{n=0}^{\infty}$ possuem propriedades semelhantes. Por conseguinte, a equação $t^{2}-a z \cdot t-a^{2}=0$ deve ter raízes com as mesmas propriedades comentadas. Nesse sentido, enunciaremos o lema 5.

Lema 5. A equação característica $t^{2}-a z \cdot t-a^{2}=0$ com raízes $\alpha(a, z)$ e $\beta(a, z)$ admite a validade, para todo inteiro $n \geq 0$, de:

$$
\left\{\begin{array}{l}
\alpha(a, \mathrm{z})^{n}=\alpha(a, \mathrm{z}) \cdot F_{n}(a, z)+a^{2} \cdot F_{n-1}(a, z) \\
\beta(a, \mathrm{z})^{n}=\beta(a, \mathrm{z}) \cdot F_{n}(a, z)+a^{2} \cdot F_{n-1}(a, z)
\end{array}\right.
$$

Demonstração: de imediato, temos que $\alpha^{2}=a z \cdot \alpha+a^{2} \cdot 1=\alpha \cdot F_{2}+a^{2} \cdot F_{1}$. Desse modo, por indução, assumiremos que $\alpha^{n-1}=\alpha \cdot F_{n-1}+a^{2} \cdot F_{n-2}, \quad$ assim, segue que $\alpha^{n}=\alpha \cdot \alpha^{n-1}=\alpha \cdot\left(\alpha \cdot F_{n-1}+a^{2} \cdot F_{n-2}\right)=\alpha^{2} \cdot F_{n-1}+\alpha \cdot a^{2} \cdot F_{n-2}=\left(a z \cdot \alpha+a^{2} \cdot 1\right) F_{n-1}+\alpha \cdot a^{2} \cdot F_{n-2}=$ $=a z \cdot F_{n-1} \cdot \alpha+a^{2} F_{n-1}+a^{2} \cdot F_{n-2} \alpha=\left(a z \cdot F_{n-1}+a^{2} \cdot F_{n-2}\right) \cdot \alpha+a^{2} F_{n-1}=F_{n} \cdot \alpha+a^{2} F_{n-1} \cdot$ Ora, determinamos que $\alpha^{n}=\alpha \cdot F_{n}+a^{2} \cdot F_{n-1}$. Mutatis mutandis, devemos determinar ainda $\beta^{n}=\beta \cdot F_{n}+a^{2} \cdot F_{n-1}$, para todo $n \geq 0$.

Finalmente, buscamos investigar uma representação da fórmula de Binnet para os Quaternions Complexos de Fibonacci, assim como sua extensão para índices inteiros. Dessa forma, vejamos o teorema 9 e o corolário 2.

Teorema 9. Os Quaternions Complexos de Fibonacci de ordem ' $n$ ', nas variáveis $a$ e $z$, admitem a seguinte fórmula variante de Binnet:

$$
Q_{n}(a, z)=\frac{\overline{\alpha(a, \mathrm{z})} \cdot \alpha(a, \mathrm{z})^{n}-\overline{\beta(a, \mathrm{z})} \cdot \beta(a, \mathrm{z})^{n}}{\alpha(a, \mathrm{z})-\beta(a, \mathrm{z})}, \text { para todo } n \geq 0,
$$

onde $\overline{\alpha(a, z)}=1+\alpha(a, z) i+\alpha(a, z)^{2} j+\alpha(a, z)^{3} k$ e $\overline{\beta(a, z)}=1+\beta(a, z) i+\beta(a, z)^{2} j+\beta(a, z)^{3} k$.

Demonstração: vamos considerar $\alpha^{n}=\alpha \cdot F_{n}+a^{2} \cdot F_{n-1}$ e $\beta^{n}=\beta \cdot F_{n}+a^{2} \cdot F_{n-1}$. Além disso, por definição, sabemos que $Q_{n}=F_{n}+F_{n+1} i+F_{n+2} j+F_{n+3} k$ para $n \geq 0$, a partir daí, segue que $\alpha \cdot Q_{n}+a^{2} \cdot Q_{n-1}=\alpha \cdot\left(F_{n}+F_{n+1} i+F_{n+2} j+F_{n+3} k\right)+a^{2} \cdot\left(F_{n-1}+F_{n} i+F_{n+1} j+F_{n+2} k\right) . \quad$ Convenientemente, 
agrupando os termos devemos obter $\alpha \cdot Q_{n}+a^{2} \cdot Q_{n-1}=\alpha F_{n}+\alpha F_{n+1} i+\alpha F_{n+2} j+\alpha F_{n+3} k+$ $a^{2} \cdot F_{n-1}+a^{2} \cdot F_{n} i+a^{2} \cdot F_{n+1} j+a^{2} \cdot F_{n+2} k=\left(\alpha F_{n}+a^{2} \cdot F_{n-1}\right)+\left(\alpha F_{n+1}+a^{2} \cdot F_{n}\right) \cdot i+\left(\alpha F_{n+2}+a^{2} \cdot F_{n+1}\right) \cdot j+\left(\alpha F_{n+3}+a^{2} \cdot F_{n+2}\right) \cdot k=$ $=\alpha^{n}+\alpha^{n+1} i+\alpha^{n+2} j+\alpha^{n+3} k=\alpha^{n}\left(1+\alpha i+\alpha^{2} j+\alpha^{3} k\right)$. Assim, reparemos que $\alpha \cdot Q_{n}+a^{2} \cdot Q_{n-1}=\alpha^{n}\left(1+\alpha i+\alpha^{2} j+\alpha^{3} k\right)$. Doravante, assumiremos que $\bar{\alpha}=1+\alpha i+\alpha^{2} j+\alpha^{3} k$. Ora, de modo análogo, obteremos $\beta \cdot Q_{n}+a^{2} \cdot Q_{n-1}=\beta^{n}\left(1+\beta \mathrm{i}+\beta^{2} j+\beta^{3} k\right) \mathrm{e}$, denotando $\bar{\beta}=\left(1+\beta \mathrm{i}+\beta^{2} j+\beta^{3} k\right)$. Logo em seguida, consideraremos o seguinte sistema:

$$
\left\{\begin{array}{l}
\alpha \cdot Q_{n}+a^{2} \cdot Q_{n-1}=\bar{\alpha} \cdot \alpha^{n} \\
\beta \cdot Q_{n}+a^{2} \cdot Q_{n-1}=\bar{\beta} \cdot \beta^{n}
\end{array}\right.
$$

e ao efetuar o cancelamento do termo repetido acima $a^{2} \cdot Q_{n-1}$, determinaremos $\alpha \cdot Q_{n}-\beta \cdot Q_{n}=$ $\bar{\alpha} \cdot \alpha^{n}-\bar{\beta} \cdot \beta^{n}$. De fato, determinamos a fórmula variante de Binnet descrita por $Q_{n}=\frac{\bar{\alpha} \cdot \alpha^{n}-\bar{\beta} \cdot \beta^{n}}{\alpha-\beta}$, para todo $n \geq 0$.

Corolário 2. Para todo inteiro ' $n$ ', vale que:

$$
Q_{-n}(a, z)=(-a)^{-n}\left(\frac{\overline{\alpha(a, \mathrm{z})} \cdot \beta(a, \mathrm{z})^{n}-\overline{\beta(a, \mathrm{z})} \cdot \alpha(a, \mathrm{z})^{n}}{\alpha(a, \mathrm{z})-\beta(a, \mathrm{z})}\right)
$$

Demonstração: pelo teorema anterior, podemos escrever $Q_{-n}=\frac{\bar{\alpha} \cdot \alpha^{-n}-\bar{\beta} \cdot \beta^{-n}}{\alpha-\beta}=\frac{\bar{\alpha} \cdot\left(\frac{1}{\alpha^{n}}\right)-\bar{\beta} \cdot\left(\frac{1}{\beta^{n}}\right)}{\alpha-\beta}=$ $=\frac{1}{(\alpha \cdot \beta)^{n}} \cdot \frac{\left(\bar{\alpha} \cdot \beta^{n}-\bar{\beta} \cdot \alpha^{n}\right)}{(\alpha-\beta)}=\frac{1}{(-a)^{n}}\left(\frac{\bar{\alpha} \cdot \beta^{n}-\bar{\beta} \cdot \alpha^{n}}{\alpha-\beta}\right)$, assim, $\quad$ obteremos $Q_{-n}=(-a)^{-n}\left(\frac{\bar{\alpha} \cdot \beta^{n}-\bar{\beta} \cdot \alpha^{n}}{\alpha-\beta}\right)$ para qualquer inteiro $n$.

Observamos que os resultados discutidos nesta seção envolvem novas noções deduzidas a partir da nova definição dos Quaternions Complexos de Fibonacci de ordem $n$, nas variáveis $a$ e $z$. À vista disso, vejamos a seguinte tabela:

Tabela 1 - Quadro resumido das identidades discutidas.

\begin{tabular}{|c|}
\hline Generalização da identidade de Cassini \\
\hline$f_{n+1} f_{n-1}-f_{n} \cdot f_{n}=(-1)^{n}$, para $n \geq 0$. \\
Giovani Domenico Cassini $(1625-1712) /$ Robert Simson $(1687-1768)$ \\
\hline$F_{n+1}(a, z) \cdot F_{n-1}(a, z)-F_{n}(a, z)^{2}=(-1)^{n} a^{2 n-2}$, para $n \geq 1 .[24]$ \\
\hline$F_{-n+1}(a, z) F_{-n-1}(a, z)-F_{-n}(a, z)^{2}=(-1)^{-n} a^{-2 n-2}$, para ' $n$ ' inteiro qualquer. \\
\hline$\left(f_{n+1}(z) f_{n-1}(z)-f_{n}(z) f_{n}(z)\right)=(-1)^{n}$ para $m \geq n \geq 1$. \\
\hline Generalização da identidade de d'Ocagne \\
\hline$f_{m+1} f_{n}-f_{m} f_{n+1}=(-1)^{m} f_{n-m}$, para $m \geq n \geq 1$. \\
\hline
\end{tabular}




\begin{tabular}{|c|c|c|}
\hline \multicolumn{3}{|c|}{ Philbert Maurice d'Ocagne (1862-1938) } \\
\hline \multicolumn{3}{|c|}{$F_{n+1}(a, z) \cdot F_{m}(a, z)-F_{n}(a, z) \cdot F_{m+1}(a, z)=(-1)^{n} a^{2 n} \cdot F_{m-n}(a, z)$, com $m \geq n \geq 1$. [24] } \\
\hline \multicolumn{3}{|c|}{$F_{-n+1}(a, z) F_{-m}(a, z)-F_{-n}(a, z) F_{-m+1}(a, z)=(-1)^{-m-1} a^{-2 m} F_{m-n}(a, \mathrm{z})$, para inteiros quaisquer. } \\
\hline \multicolumn{3}{|c|}{$f_{n+1}(z) \cdot f_{m}(z)-f_{n}(z) \cdot f_{m+1}(z)=(-1)^{n} \cdot a^{n-m+1} \cdot f_{m-n}(z)$, para $m \geq n \geq 1$} \\
\hline \multicolumn{3}{|c|}{ Generalização da identidade de Catalan } \\
\hline \multicolumn{3}{|c|}{$\begin{array}{c}f_{n} f_{n}-f_{n+r} f_{n-r}=(-1)^{n-r} f_{r}^{2}, m \geq n \geq 1 . \\
\text { Eugène Charles Catalan }(1814-1894)\end{array}$} \\
\hline \multicolumn{3}{|c|}{$F_{n}(a, z) F_{m}(a, z)-F_{n-r}(a, z) F_{m+r}(a, z)=(-1)^{n-r} a^{2 n-2 r} \cdot F_{m-n+r}(a, z) \cdot F_{r}(a, z)$, para $m \geq n \geq 1 .[24]$} \\
\hline \multicolumn{3}{|c|}{$F_{-n}(a, z) \cdot F_{-m}(a, z)-F_{-n-r}(a, z) \cdot F_{-m+r}(a, z)=(-1)^{-m+r} a^{-2 m+2 r} \cdot F_{m-n-r}(a, z) \cdot F_{-r}(a, z)$, para inteiros quaisquer. } \\
\hline \multicolumn{3}{|c|}{$\left(f_{n}(z) \cdot f_{m}(z)-f_{n-r}(z) \cdot f_{m+r}(z)\right)=(-1)^{n-r} \cdot a^{n-m-2 r+2} \cdot f_{m-n+r}(z) \cdot f_{r}(z)$, para $m \geq n \geq 1$} \\
\hline \multicolumn{3}{|c|}{ Generalização da Fórmula de Honsberger } \\
\hline \multicolumn{3}{|c|}{$\begin{array}{c}f_{n+m+1}=f_{n+1} f_{m+1}+f_{n} f_{m} \\
\text { Ross Honsberger }(1929-2016)\end{array}$} \\
\hline \multicolumn{3}{|c|}{$F_{n+m+1}(a, z)=F_{n+1}(a, z) F(a, z)_{m+1}+a^{2} \cdot F_{n}(a, z) \cdot F_{m}(a, z)$, para $n, m \geq 0 .[24]$} \\
\hline \multicolumn{3}{|c|}{$F_{-n-m+1}(a, \mathrm{z})=F_{-n+1}(a, z) F_{-m+1}(a, z)+a^{2} \cdot F_{-n}(a, z) F_{-m}(a, z)$, para inteiros quaisquer. } \\
\hline \multicolumn{3}{|c|}{$f_{n+m+1}(z)=f_{n+1}(z) f_{m+1}(z)+f_{n}(z) \cdot f_{m}(z)$, para $n, m \geq 0$. } \\
\hline \multicolumn{3}{|c|}{ Quaternions Complexos de Fibonacci de ordem ' $n$ ' } \\
\hline \multicolumn{3}{|c|}{$F_{n}(a, z)=a \mathrm{z} F_{n-1}(a, z)+a^{2} F_{n-2}(a, z)$, para $n \geq 2$} \\
\hline \multicolumn{3}{|c|}{$Q_{n+1}(a, z)=a z \cdot Q_{n}(a, z)+a^{2} Q_{n-1}(a, z)$, para $n \geq 0$} \\
\hline \multicolumn{3}{|c|}{$Q_{-n}(a, z)=(-1)^{-n-1} a^{-2 n}\left(F_{n}(a, \mathrm{z})-a^{2} F_{n-1}(a, \mathrm{z}) \cdot i+a^{4} F_{n-2}(a, \mathrm{z}) \cdot j-a^{6} F_{n-3}(a, \mathrm{z}) \cdot k\right), \quad \forall n \in \mathbb{Z}}$. \\
\hline \multicolumn{3}{|c|}{$Q_{n}(a, z)=\frac{\overline{\alpha(a, \mathrm{z})} \cdot \alpha(a, \mathrm{z})^{n}-\overline{\beta(a, \mathrm{z})} \cdot \beta(a, \mathrm{z})^{n}}{\alpha(a, \mathrm{z})-\beta(a, \mathrm{z})}, \forall n \geq 0$} \\
\hline \multirow[t]{2}{*}{$Q_{-n}(a, z)=(-a)^{-n}$} & $\left.\overline{\alpha(a, \mathrm{z})} \cdot \beta(a, \mathrm{z})^{n}-\overline{\beta(a, \mathrm{z})} \cdot \alpha(a, \mathrm{z})^{n}\right)$ & \multirow[t]{2}{*}{$\forall n \in \mathbb{Z}$} \\
\hline & $\alpha(a, \mathrm{z})-\beta(a, \mathrm{z})$ & \\
\hline
\end{tabular}

Fonte: elaborada pelos autores.

A tabela 1 apresenta algumas identidades que foram discutidas neste artigo, com isso podese compreender, numa perspectiva epistemológica, aspectos históricos e evolutivos do modelo de Fibonacci, no que diz respeito a sua extensão para índices inteiros e complexificação com a inserção das unidades imaginárias e variáveis complexas.

\section{Conclusão}

No presente trabalho discutimos propriedades e identidades generalizadas intimamente relacionadas com o modelo de Fibonacci, segundo uma representação particular na variável complexa [1,2]. Nossa discussão foi desenvolvida a partir do trabalho [16] que abordam outro interesse, na medida em que, desenvolvem várias propriedades dos polinômios de HOMFLY, que se caracterizam como uma classe de funções polinomiais na variável complexa que é um estudo relevante dentro da Teoria dos Nós. 
Segundo a discussão desenvolvida em [16], os polinômios de HOMFLY constituem uma generalização concebida por Alexander Horton Conway (1937 - ?) e o matemático norte americano James Waddell Alexander (1888 - 1971).

Certamente que outras implicações podem ser derivadas, todavia, restringimo-nos numa apreciação de propriedades que se mostram indissociavelmente vinculadas ao modelo da sequência de Fibonacci. Nesse sentido, discutimos a extensão das identidades de Cassini, d'Ocagne, Catalan a partir de índices inteiros quaisquer, posto que não registramos sua discussão no trabalho indicado em [16]. Além disso, exploramos a fórmula (generalizada) de Honsberger. Dessa forma, vislumbramos para essas identidades a possibilidade do processo de extensão contínuo para outros conjuntos numéricos.

Além do mais, abordamos também algumas propriedades originadas da noção de Quaternions Complexos de Fibonacci de ordem ' $n$ ', nas variáveis $a$ e $z$, como também os elementos definidos pela seguinte recorrência $Q_{n}(a, z)=F_{n}(a, z)+F_{n+1}(a, z) i+F_{n+2}(a, z) j+F_{n+3}(a, z) k$, para um índice inteiro qualquer. Vale acentuar seu caráter de ineditismo que proporcionamos para o debate científico (tabela 1).

Ademais, o processo de extensão das identidades mencionadas [4, 5], no campo de índices inteiros quaisquer, não é discutido nos trabalhos consultados, assim como sua representação correspondente na variável complexa intimamente vinculada ao modelo de Fibonacci.

Finalmente, na tabela 1, trazemos um quadro resumido dos resultados principais e das identidades obtidas ao decurso do texto. Reparemos, todavia, que assinalamos o processo de generalização, quanto à complexificação, das identidades de Cassini, de d'Ocagne e da identidade generalizada de Catalan (teoremas 4, 6 e 7).

\section{Referências}

1. ALVES, F. R. V. Sobre a evolução matemática, histórico-epistemológica do modelo de Fibonacci: sobre a abordagem matricial. Revista THEMA, v. 14, n. 1, p. 91-111, 2017.

2. ALVES, F. R. V. Sequência Generalizada de Pell (SGP): aspectos históricos e epistemológicos sobre a evolução de um modelo. Revista THEMA, v. 13, n. 2, p. 27-41, 2016a.

3. ALVES, F. R. V. Descobrindo definições matemáticas no contexto de investigação histórica: o caso da sequência generalizada de Fibonacci. BOLETIM GEPEM, n. 68, p. 112$117,2016 b$.

4. ALVES, F. R. V. Engenharia didática para a generalização da sequência de Fibonacci: uma experiência num curso de licenciatura. Educação Matemática Pesquisa, v. 18, n. 1, p. 61-93, 2016c.

5. ALVES, F. R. V. Sobre a evolução histórica do modelo de Fibonacci: a classe das funções hiperbólicas de Fibonacci - FHF. Revista VIDYA, v. 35, n. 1, p. 133-146, 2015.

6. JORDAN, J. H. Gaussian Fibonacci and Lucas Numbers. The Fibonacci Quarterly, v. 3, n. 4 , p. $315-318,1965$.

7. HONSBERGER, R. Mathematical gems III. Washington: Mathematical Association of America, 1985. (The Dolciani Matematical Exposition, v. 9). 
8. HOGGATT, V. E.; LONG, C. T. Divisibility properties of generalized Fibonacci polynomials. The Fibonacci Quarterly, v. 12, n. 2, p. 113-120, 1974.

9. HOGGATT, V. E.; BICKNELL, M. Generalized Fibonacci Polynomials. The Fibonacci Quarterly, v. 11, n. 5, p. 457-465, $1973 \mathrm{a}$.

10. IAKIN, A. L. Generalized quaternions with quaternion components. The Fibonacci Quarterly, v. 15, n. 4, p. 350-352, 1977.

11. IAKIN, A. L. Extended Binet forms for generalized quaternions of higher order. The Fibonacci Quarterly, v. 19, n. 5, p. 410-413, 1981.

12. KOSHY, T. Elementary number theory and applications. 2. ed. Boston: Elsevier, 2007.

13. KING, C. H. Conjugate generalized Fibonacci sequences. The Fibonacci Quarterly, v. 6, n. 1, p. 46-50, 1968.

14. PLAZA, A.; FALCÓN, S. Combinatorial proofs of Honsberger-type identities.

International Journal of Mathematical Education in Science and Technology, v. 39, n. 6, p. 785-792, 2008.

15. SMITH, D. E. A source book in Mathematics. New York: Dover, 1929, v. 1.

16. TASKOPRÜ, K.; ALTINTAS, Î. HOMFLY polynomials of torus links as generalized Fibonacci polynomials. The Electronic Journal of Combinatorics, v. 22, n. 4, p. 1-17, 2015.

17. GARTH, D.; MILLS, D.; MITCHELL, P. Polynomials generated by the Fibonacci Sequence. Journal of Integer Sequences, v. 10, n. 6, 1-12, 2007.

18. GOULD, H. W. A history of the Fibonacci q-matrix and a higher-dimensional problem. The Fibonacci Quarterly, v. 19, n. 3, 250-257, 1981.

19. GRIMALDI, R. P. Fibonacci and catalan numbers. New Jersey: John Wiley \& Sons, Inc., 2012.

20. HOGGATT, V. E.; BICKNELL, M. A primer for the Fibonacci Numbers: part XIV. The Fibonacci Quarterly, v. 12, n. 2, p. 147-156, 1974.

21. KAUERS, M.; PAULE, P. The concrete tetrahedron: symbolic sums, recurrence equations, generating functions, asymptotic estimates. New York: Springer, 2011.

22. KOSHY, T. Fibonacci and Lucas numbers and applications. New York: John Willey and Sons, 2011.

23. SWAMY, M. N. Futher properties of Morgan Voyce polynomials. The Fibonacci Quarterly, v. 6, n. 2, p. 167-176, 1968. 
24. SWAMY, M. N. S. On generalized Fibonacci quaternions. The Fibonacci Quarterly, v. 11, n. 5, p. 547-549, 1973.

25. WEB, W. A.; PARBERRY, E. A. Divisibility properties of Fibonacci polynomials. The Fibonacci Quarterly, v. 7, n. 5, p. 457-463, 1969.

26. WHITFORD, A. K. Binet's formula generalized. The Fibonacci Quarterly, v. 15, n. 1, p. 21/24/29, 1977.

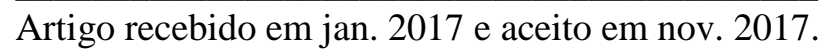

